\title{
Violence urbaine, démocratie et changement culturel : l'expérience brésilienne (Partie 4)
}

\section{Angelina Peralva}

\section{OpenEdition}

1 Journals

\section{Édition électronique}

URL : http://journals.openedition.org/conflits/710

DOI : $10.4000 /$ conflits. 710

ISSN : $1777-5345$

Éditeur :

CCLS - Centre d'études sur les conflits lilberté et sécurité, L'Harmattan

Édition imprimée

Date de publication : 15 octobre 1998

ISSN : 1157-996X

Référence électronique

Angelina Peralva, «Violence urbaine, démocratie et changement culturel : l'expérience brésilienne (Partie 4) », Cultures \& Conflits [En ligne], 29-30 | automne-hiver 1998, mis en ligne le 16 mars 2006, consulté le 30 mars 2021. URL : http://journals.openedition.org/conflits/710 ; DOI : https://doi.org/ $10.4000 /$ conflits. 710

Ce document a été généré automatiquement le 30 mars 2021.

Creative Commons License 


\title{
Violence urbaine, démocratie et changement culturel : l'expérience brésilienne (Partie 4)
}

\author{
Angelina Peralva
}

Ces transformations sont particulièrement sensibles chez les jeunes des milieux populaires, pour lesquels les changements que nous venons d'indiquer se sont opérés de manière rapide et brutale. Elles s'inscrivent doublement, dans l'affaiblissement des liens tissés avec un lieu particulier d'appartenance, et dans la transformation des rapports entretenus avec la société en général. patrons et les travailleurs et doublé cette séparation d'une autre, entre pauvres et riches. Même là où la représentation industrielle du travail était faible, les travailleurs tiraient leur fierté et construisaient leur identité en s'appuyant sur les valeurs diffusées par l'expérience du travail en général. Les liens avec la société se tissaient sur cette base, d'autant plus cordiaux et perçus comme tels, que les conflits du travail n'avaient pas une place centrale - ce qui a toujours été le cas à Rio de Janeiro, capitale de la République et ville de fonctionnaires jusqu'en 1961, puis ville de prestation de services, et capitale médiatique du pays jusqu'à aujourd'hui.

4 Le fait que le travail structurait autour de lui le lien principal entre les milieux populaires et la société en général avait comme pendant le relatif isolement de ces milieux à d'autres niveaux. Les formes de sociabilité, les modalités de loisir ne se mélangeaient pas, elles s'inscrivaient à l'intérieur d'univers ségrégués. Dans le cas de Rio de Janeiro, cette constatation est particulièrement frappante dans la mesure où la ville offre tout au long de l'année à ses habitants une forme très prisée de loisir qui est la plage. Or, l'investissement massif de la plage par des jeunes des milieux populaires est un fait relativement récent, et dont la visibilité s'est accrue de manière spectaculaire depuis la redémocratisation. 
5 Spatialement, on peut grossièrement partager la ville de Rio en deux grandes zones : nord et sud. La zone sud correspond aux quartiers des plages, progressivement occupés par les couches aisées de la population à partir des années quarante. Auparavant, la ville se déployait vers le nord. Une plage très polluée dans le côté nord a été longtemps fréquentée par la population pauvre des environs. Le grand changement est lié, dans les années quatre-vingt, à la démocratisation de l'accès aux plages par une modification volontaire de la politique des transports et la création de lignes directes d'autobus reliant la zone nord à la zone sud. Cette explication est néanmoins insuffisante pour éclairer la nouvelle tendance à un investissement des plages des quartiers riches, pendant le week end, par la population des quartiers pauvres et notamment par les jeunes. Nous avons interrogé de vieux habitants d'une favela de la zone sud, pour lesquels l'indisponibilité des transports n'a jamais été un obstacle interdisant l'accès à la plage, puisqu'ils en étaient si proches qu'ils pouvaient y accéder même à pied. Ils nous ont dit que dans le passé, aller à la plage n'était important, ni pour eux, ni pour leurs enfants. Les week-ends étaient passés en famille, dans la favela même, et autour d'activités de loisir et de formes de sociabilité propres à ce milieu.

6 L'investissement de la plage par les jeunes favelados doit être compris à l'intérieur d'un double mouvement de transformation du rapport entretenu par les couches populaires avec la société en général. D'un côté, l'affaiblissement du travail en tant qu'élément principal de structuration de ce lien; de l'autre, la démocratisation des formes de loisir, qui concernent aujourd'hui de plus en plus les jeunes en général, plutôt que des jeunes strictement définis par un milieu social d'appartenance. D'un côté, les formes de loisir autrefois exclusives des couches moyennes, comme la plage, deviennent un espace plus démocratique et un lieu de mélange de jeunes issus de différents milieux; de l'autre, les formes les plus actuelles de loisir développées dans les milieux populaires, les bals funk à Rio, le monde du rap à São Paulo, attirent des jeunes issus des couches moyennes, qui s'y mêlent pour leur part. Si cela a été possible, c'est justement parce que les jeunes des milieux populaires deviennent de plus en plus des jeunes tout court, et non plus, comme dans le passé, de jeunes travailleurs. Lorsqu'ils travaillent, le rapport au travail prend rarement l'ancienne forme classique du travail salarié industriel à temps complet - et même travailleurs, ils sont en même temps des collégiens, des lycéens ou des étudiants, ainsi que des consommateurs de mode, de musique et de loisirs. Malgré les défaillances dans l'offre des politiques publiques au Brésil, leur niveau de scolarité s'est considérablement élevé par rapport à celui de leurs parents, la qualité de leur vie s'est améliorée et leur niveau de consommation s'est accru.

7 Cette déségrégation est d'autant plus significative, dans le cas de Rio de Janeiro, qu'elle ne correspond pas, dans cette ville, à un bouleversement dans les formes d'inscription spatiale des populations pauvres, qui continuent à habiter les mêmes quartiers et les mêmes favelas que dans le passé ; de plus, la présence de nombreuses favelas au milieu des quartiers bourgeois de la zone sud de Rio n'a jamais été réellement perçue comme gênante, puisque, tout en étant là, elles étaient de fait quasiment invisibles au regard extérieur ${ }^{1}$. Dès lors, la déségrégation à laquelle nous nous référons est un fait symbolique. L'invisibilité des populations pauvres s'est évanouie, et cette déségrégation a entraîné tout un lot de violences, auparavant inconnues.

8 Nous reviendrons sur les formes et sur les raisons de cette violence, s'inscrivant dans le processus de déségrégation auquel nous nous sommes référé. Pour l'instant, il convient d'ajouter qu'à la déségrégation a correspondu une dynamique de reségrégation, parmi 
les couches moyennes et les élites, mais ayant aussi, comme nous le verrons, son propre pendant dans les milieux populaires. A São Paulo, les logiques de reségrégation ont profondément modifié le panorama urbain ${ }^{2}$. Les nouveaux murs dressés pour rétablir la séparation entre les strates sociales ne sont plus seulement symboliques, ils sont matériels et physiques. Des bouleversements dans le marché de l'immobilier ont entraîné la construction de grandes résidences fermées dans la région métropolitaine, censées constituer des espaces protégés où les parents des milieux aisés seraient à même d'élever leurs rejetons. Ces transformations se sont aussi produites à Rio de Janeiro. Dans les deux villes, elles ont laissées des marques plus modestes et minuscules avec les grillages qui entourent désormais les simples immeubles d'habitation, autrefois ouverts sur la rue ; dans la présence permanente des concierges, 24 heures sur 24, isolés dans des cages spéciales qui leur sont destinées, d'où ils peuvent voir ceux qui s'approchent, tout en restant à l'abri d'éventuelles agressions, communiquant par interphone avec la rue et faisant le tri parmi ceux qui veulent accéder à l'immeuble.

Ce barrage matériel, reste, dans le cas de Rio en tout cas, bien faible face à l'ampleur de la déségrégation symbolique. Les enfants des favelas sont de plus en plus nombreux à fréquenter des écoles privées, auxquelles ils accèdent souvent grâce à des bourses d'étude. De même que la plage, les écoles sont des lieux où se tissent des amitiés juvéniles, et c'est surtout par ce biais que les murs de la reségrégation sont franchis. Dès lors, on fréquente les mêmes discothèques, ou encore les salles de bals où, dans les immeubles des couches moyennes, les adolescents organisent leurs " booms ". Cependant, s'il est vrai que l'interpénétration des univers de la favela et des couches moyennes est un fait réel, cela s'est généralement effectué dans le sens de la favela vers la ville, plutôt que dans le sens inverse. Certes, la capacité du jeune favelado à se lier d'amitié avec d'autres jeunes, en dehors de son milieu d'origine, s'est accrue. Il est par contre bien plus rare que des jeunes issus des couches moyennes viennent dans une favela rendre visite à un ami. Se déplacer dans une favela reste pour eux du domaine de la transgression. On vient y acheter de l'herbe, lorsqu'on en consomme; pour les bals funk, pendant le week end - et là, on prend un risque, car les bals funk sont des lieux où les débordements violents sont toujours possibles.

L'épuisement de la politique et le discours social de la criminalité

11 Le processus de déségrégation, et notamment l'entrée des jeunes des milieux populaires dans la société de masse, ne dérivent pas seulement d'une élévation générale de leur niveau de vie, ce qui est un fait réel (Peralva, 1996). Il a été préparé par les mouvements populaires qui, dès la fin des années soixante-dix, s'étaient lancés dans une lutte pour la redémocratisation du pays. On peut dire que les mouvements sociaux ont constitué une première forme, politique, de ce processus de déségrégation qui apparaît aujourd'hui plus nettement à nos yeux. Dans les favelas de Rio de Janeiro, ils ont été au coeur de la lutte pour l'urbanisation, laquelle a par ailleurs produit des effets remarquables, du point de vue de l'amélioration de la qualité de la vie.

Dès le milieu des années quatre-vingt, on observe toutefois un essoufflement, qui va toucher de manière généralisée l'ensemble des luttes populaires et des mouvements syndicaux. Cet essoufflement s'inscrit dans la fragmentation des luttes sociales, due au passage à la démocratie, et à l'affaiblissement de l'adversaire - la dictature militaire auquel pendant de longues années les mouvements sociaux s'étaient opposés (opposition dont ils avaient tiré leur fierté et leur force). Il s'inscrit aussi dans une conjoncture marquée par la crise économique et par la désynchronisation des 
politiques publiques au niveau de l'union, des Etats et des communes - contexte dans lequel les mouvements syndicaux ont essuyé plusieurs défaites, sans pouvoir contrecarrer (au moins dans certaines branches) une tendance générale à la baisse des revenus de leurs adhérents ${ }^{3}$.

Les causes de cet essoufflement semblent plus complexes s'il s'agit des luttes urbaines. Il ne se doit pas seulement à des défaites, qui ont eu lieu comme ailleurs, mais tout autant à leur succès. C'est du moins ce que suggère l'analyse de l'expérience d'une favela que nous étudions à Rio. Le succès s'inscrit dans un mouvement d'urbanisation réel de la favela - amélioration de la qualité et des disponibilités en matière d'électricité et d'eau courante, collecte d'ordures ménagères, pénétration du réseau téléphonique, une vraie révolution sur le plan des conditions matérielles de vie. L'échec tient à l'incomplétude de l'urbanisation, aux projets abandonnés, aux innombrables promesses non tenues par les responsables des politiques de la ville. Mais surtout, les luttes urbaines s'étaient développées sous la forme d'actions collectives, qui s'étaient par la suite converties en structures de prise en charge de l'urbanisation par les favelados eux-mêmes, depuis l'intérieur des favelas. Les militants ont toujours souhaité qu'il en soit ainsi, et l'ensemble du processus d'urbanisation a été négocié, puis son application concrète gérée en régime de partenariat. Cette mobilisation collective s'est essoufflée, autant en raison des succès de la lutte (dans la mesure où plusieurs revendications ont été acquises) qu'en raison de ses échecs.

L'essoufflement coöncide par ailleurs avec la montée de la criminalité et du trafic de drogue, et l'un comme l'autre s'inscrivent dans le même mouvement de transformation. Dès lors, le discours social de la criminalité remplace d'une certaine manière le discours politique, ce qu'on peut expliquer de deux manières. D'un côté, il s'inscrit dans le vide du discours politique, un vide qui n'est pas seulement celui d'une modalité particulière d'action, mais celui du mouvement pour la redémocratisation en général. De l'autre, il opère en quelque sorte en symbiose avec un univers social qui reste en partie ségrégué, et dont la criminalité contribuera elle-même à accroitre la ségrégation, un peu comme le pendant des élites étudiées par Teresa Caldeira, lesquelles, face aux transformations de la société de masse, ont élevé des murs dans la ville ${ }^{4}$.

Cette symbiose entre le bandit et le milieu social dans lequel il s'appuie n'est pas sans rapports avec le phénomène du bandit social étudié par Eric Hobsbawm ${ }^{5}$. Elle s'inscrit dans l'expérience d'une illégalité, qui est aussi une illégitimité, partagée, et par la population et par le bandit. Ce dernier, par la radicalité de l'action illégale dans laquelle il se trouve engagé, et aussi parce qu'il fait l'objet d'une persécution plus ouverte par les forces de l'ordre (dans le cas de Rio, un ordre toujours perçu comme extérieur aux segments pauvres de la population), finit par apparaître comme une sorte de porteparole de tous. Il ne s'agit pas pour nous d'accentuer indûment les aspects les plus romantiques de cette symbiose - il $\mathrm{y}$ a des cas où le bandit s'assure par la terreur pure et simple l'appui, même passif, du milieu dans lequel il est inscrit. Mais pourtant, ce rapport est souvent réellement et remarquablement géré par les bandits, de sorte qu'ils apparaissent comme les porte-paroles d'une opposition entre pauvres et riches.

Une nouveauté par rapport aux bandits sociaux traditionnels, décrits par Hobsbawm, est que la symbiose dont il s'agit n'est pas seulement sociale, elle est sociogénérationnelle. Encore une fois ici, l'opposition entre les générations intervient en tant que catégorie permettant de décrire la différenciation de l'expérience d'adultes et 
jeunes. Malgré l'élévation du niveau de scolarité de leurs enfants, les profondes transformations subies par le marché de l'emploi limitent pour les familles faveladas les possibilités de formuler des propositions de métiers futurs pour leurs enfants. En revanche, elles savent parfaitement ce qu'elles refusent à leur progéniture. Avant tout, elles ne veulent pas que l'enfant s'engage dans le trafic des drogues. Dans une formulation un peu plus positive, elles souhaiteraient que l'enfant soit " quelqu'un ", c'est-à-dire, le contraire d'un bandit.

Ces valeurs, affirmées au sein de la famille, sont néanmoins relativisées par le mode à travers lequel se structurent les relations entre la favela et la ville. Le regard de la ville, englobant l'ensemble des favelados dans l'expérience de la marginalité et du crime, affaiblit l'effort de clivage entre marginaux et non marginaux, entrepris dans la sphère familiale, et relativise sa signification. Ceci est d'autant plus important au moment de l'adolescence, dans la mesure où l'adolescent construit sa propre identité à travers l'identification aux pairs. D'un côté, il revendique, face à la famille, une autonomie de jugement et le droit à l'autodétermination. De l'autre, la " juvénisation " du trafic des drogues a une incidence directe sur le flou dont sont imprégnés les rapports entre jeunes trafiquants et non trafiquants. A l'intérieur de la favela, ils partagent une histoire commune. Ils partagent aussi une expérience générationnelle commune, à travers l'accroissement des niveaux de scolarité, qui vaut pour l'ensemble des jeunes et qui n'exclut pas le bandit; à travers l'accroissement des niveaux de participation culturelle et des formes communes d'insertion dans la société de masse - mais aussi à travers la définition négative de la favela, cernée dans le regard extérieur, à travers l'importance du préjugé contre le favelado et de la violence, en tant qu'élément structurant le rapport entre la favela et la ville. Les fêtes propres à la favela, et surtout les bals funk, marquent de leur sceau cette complicité. En ce sens, le discours social de la criminalité est le pendant de la désaffection à l'égard de la politique et des politiciens qu'on retrouve à l'heure actuelle chez les jeunes favelados de Rio de Janeiro.

Déstabilisation identitaire et violence des jeunes

Reste à expliquer, non plus maintenant du point de vue de l'effondrement de l'Etat dans sa capacité à contrôler la violence, mais du point de vue de l'entrée dans la société de masse, pourquoi celle-ci favorise, chez les jeunes surtout, le développement d'une disponibilité à la violence.

Trois éléments sont à prendre en considération. Premièrement, l'indétermination d'un rapport au monde (qui semble de moins en moins défini en termes sociaux et de plus en plus en termes de mobilisation de ressources personnelles), ainsi que la généralisation de la violence dans la société, induisent de façon complémentaire une perception de l'environnement en termes de risques, suscitant presque naturellement des réponses en termes de conduites de risque. Deuxièmement, la déstabilisation identitaire des jeunes issus des milieux populaires les amène à une définition du monde en termes de rapports de force. Enfin, le marché des stupéfiants, qui a fonctionné ces dernières années en tant qu'opérateur du changement, apparaît comme une voie d'entrée dans la violence.

Risque et conduites de risque

Le risque apparaît aujourd'hui comme une dimension générale de l'expérience contemporaine. Au fur et à mesure que la société de masse se développe et se démocratise, le poids relatif du choix individuel s'accroit, comparativement à celui de la destinée sociale. Ce choix concerne la vie des individus; il se fonde sur la 
mobilisation de ressources personnelles et apparemment transforme l'individu en seul maitre de son propre succès ou de ses propres échecs. Evidemment l'importance objective des déterminations sociales reste entière et, dans le domaine de l'école par exemple, les théories de la reproduction l'ont suffisamment démontré pour qu'il ne soit pas nécessaire d'y revenir. La démocratisation de l'accès à l'école et à d'autres espaces sociaux produit néanmoins un effet de bouleversement des perceptions à propos de la place de chaque individu dans le monde. Maintes représentations qui régulent la vie des élites, l'idée de dynamisme individuel, l'idée qu'il faut oser, sont empreintes de cette représentation qui veut que la prise de risque et le succès personnel sont les deux faces de la même monnaie.

Moins une société est socialement protégée, plus ces représentations ont tendance à se diffuser vers les couches populaires, plus grandes deviennent les chances de prises de risque non maîtrisées et associées à des conduites d'autant plus violentes que la violence, justement, compense l'absence de maîtrise. Dans la société brésilienne, où la violence s'est élargie ces dernières années à l'ensemble du corps social, la prise individuelle d'un risque, et d'un risque de mort, est presque une réponse naturelle à la généralisation de l'expérience de la mort violente sur l'ensemble de la société.

Nous avons déjà évoqué ce problème lorsque nous avons traité de l'impact des escadrons de la mort sur l'accroissement de la violence liée à la criminalité. Face au risque d'être tué, le voleur devient lui-même un tueur. Et il le devient d'autant plus qu'il sait que toute la société est en train de s'armer. En ce sens, la gestion du risque par la prise de risque participe, bien évidemment, à la spirale de la violence.

Ce phénomène a, dans la société brésilienne, bien d'autres expressions. Il suffit de rappeler le refus des conducteurs de s'arrêter aux feux rouges: au risque de se faire attaquer, on préfère celui de l'accident, face auquel on a le sentiment d'une plus grande maitrise, que face à une attaque à main armée. Il a induit chez les jeunes une modalité particulièrement intéressante de prise de risque, le " surf du rail ". Plutôt que de voyager à l'intérieur, ce qui non seulement est perçu comme moins confortable, mais tout autant comme porteur de risques, certains jeunes préfèrent effectuer le voyage audessus du toit des trains. La dégradation des trains urbains n'est pas seulement sensible à travers le délabrement des wagons qui circulent sur certaines lignes, mais aussi par leur suroccupation, ce qui s'explique en fonction du nombre insuffisant des trains en circulation, et de la présence constante de voleurs qui dévalisent les voyageurs. Compte tenu des désavantages comparatifs d'un voyage à l'intérieur des wagons, ou au-dessus des compositions, de nombreux jeunes penchent vers la seconde solution.

Disponibilité à la violence

Les favelas de Rio de Janeiro constituent aujourd'hui un observatoire particulièrement significatif des problèmes de déstabilisation identitaire que nous sommes en train d'évoquer. Contrairement à celles de São Paulo, souvent bâties sur des terrains plats et plus facilement intégrables à l'environnement urbain, celles de Rio de Janeiro sont la plupart du temps installées sur des collines, la topographie accidentée favorisant leur maintien sous cette forme originale et leur isolement relatif par rapport à l'environnement. Certaines, dans le centre ville, ou dans la zone nord, datent de la fin du siècle dernier; celles de la zone sud, plus récentes, datent des années quarante. Leur installation a accompagné le mouvement d'urbanisation de ces quartiers. Il ne s'est jamais agi de lieux de transit, mais d'un véritable habitat populaire traditionnel, où les générations se succèdent, où des héritages se transmettent, un lieu aussi de production 
culturelle dont les expressions les plus connues sont les écoles de samba et le carnaval. Dès lors, le favelado était un travailleur, au sens le plus universel de cette définition; il était aussi un producteur culturel accepté et légitimé par la ville. Ces deux faces de la vie dans la favela la marquaient d'un signe positif, malgré la pauvreté de l'habitat.

Depuis quelques années déjà, et en particulier avec la redémocratisation, ces deux éléments se sont affaiblis dans leur capacité à définir une identité collective et un lien avec la ville. Le travail, en fonction des transformations que nous avons évoquées. Quant aux écoles de samba, les changements qui les touchent sont encore plus anciens. Institutionnalisées par la ville, complètement intégrées à l'industrie du tourisme et du carnaval, elles ne constituent plus un lieu central de mobilisation de la jeunesse, laquelle s'est davantage retournée vers une production musicale internationalisée - ce qui se manifeste dans son engouement pour les bals funk.

D'une certaine manière, les bals funk occupent, pour ce qui est de la population juvénile des favelas, la place auparavant prise par les écoles de samba dans l'expérience des générations précédentes. Ils sont un lieu d'identification collective des jeunes favelados, qui par ailleurs attire, comme nous l'avons dit, un petit nombre d'autres jeunes, issus des couches moyennes. Ce mode d'identification, néanmoins, ne suffit pas à lui seul à combler le vide laissé par l'affaiblissement du travail en tant qu'élément constitutif de l'identité individuelle et collective. Le jeune favelado devient de plus en plus un jeune tout court, indifférencié face à la société de masse, et un favelado. Dès lors, la condition de favelado assume un sens négatif, qu'elle n'avait pas nécessairement dans le passé, ou qui, dans le passé, pouvait être plus aisément relativisé.

30 La race, ou plus exactement le fait d'être noir, ce qui est souvent le cas dans les favelas, émerge par ailleurs comme une difficulté supplémentaire du point de vue du processus de construction de la personnalité individuelle. Dissous dans le passé, derrière une thématique sociale générale, ce problème jaillit maintenant d'une manière autrement importante. Pour les adolescents des favelas, il est aujourd'hui particulièrement difficile de gérer leur rapport à un milieu social autre que le leur, en étant et favelado et noir. Sous l'emprise de la culture de masse, leur idéal-type d'homme ou de femme désirés s'inspire toujours d'un acteur des feuilletons télévisés - blanc, blond, aux yeux clairs. Le retard des media à construire l'image du noir comme un type positif de beauté distord pour l'adolescent des favelas, lui même souvent noir, les matrices d'identification à travers lesquelles il se projette dans un partenaire idéal.

31 Le préjugé contre le favelado et contre le noir, ancré dans une tradition de longue date, mais autrefois compensé par d'autres liens positifs qui s'établissaient entre la favela et la ville, est aujourd'hui, pour le jeune, plus difficile à vivre. Dès lors, son expérience se définit dans une grande mesure par la tension permanente entre son indifférenciation accrue par rapport à des jeunes d'autres milieux sociaux et cette différence négative, qui persiste et qui devient plus sensible qu'avant. Dans les conditions actuelles, elle ne peut être compensée que par la force, et elle induit chez lui une perception du monde en termes de violence et de rapports de force.

32 Aussi, c'est exactement au moment où elle se rapproche le plus de la ville, par ses conditions de vie, par l'intégration individuelle des jeunes favelados à la culture de masse et à des formes de loisirs partagées par la jeunesse de tous les milieux sociaux, que la favela va se reconstruire, à travers la violence, en tant que communauté imaginaire. En ce sens spécifique, il faut dire qu'il existe aujourd'hui dans les favelas un 
potentiel juvénile d'engagement dans la violence, qui s'inscrit dans les transformations historiques les plus générales qui touchent la société brésilienne ${ }^{6}$. surtout concernée par une large zone de frontière, impossible à contrôler avec des moyens traditionnels, en raison de son étendue même. Les stupéfiants entreposés dans les favelas de la ville, surtout de la cocaïne et de l'herbe (le crack étant un phénomène typique de São Paulo), desservent un marché intérieur situé en dehors des favelas ellesmêmes ${ }^{9}$. La crise et l'affaiblissement de la capacité de l'Etat à contrôler la violence, l'effondrement de l'institution policière, l'absence de contrôle sur le trafic international des armes favorisent cette transformation des favelas en entrepôts de drogue, susceptibles de desservir les couches moyennes et les élites de la ville. longue, définie par l'expérience d'illégalité dont cette forme d'habitat a toujours été frappé, en raison de l'occupation sauvage des terrains, de l'inexistence d'actes de propriété, bref de la définition statutaire de la favela elle-même, un lieu à l'égard duquel la politique de la ville n'a pas d'obligations clairement établies, et des menaces de déplacement qui ont de tous temps pesé sur sa population. Cette disponibilité s'inscrit aussi dans une histoire courte, définie par la place du risque et des conduites 
de risque, ainsi que par la déstabilisation identitaire dont le jeune favelado est victime, et qui peut l'induire à récupérer par la force sa place dans le monde. Sortir de la violence?

La redémocratisation a eu au Brésil des effets positifs, mais aussi beaucoup d'effets négatifs, à commencer par la crise de l'Etat, et en particulier l'effondrement de sa capacité à contrôler la violence. Parmi les effets positifs, il faut compter ceux en rapport avec l'accélération de l'intégration des couches populaires à la société de masse, leur plus grande participation à la consommation et à la culture. Contrairement à l'argumentation inconsistante qui associe la violence à un accroissement de l'exclusion $^{10}$, il faut reconnaître que la dynamique de la redémocratisation a été essentiellement marquée par l'inclusion des couches populaires, et non pas l'inverse.

1 L'intégration des couches populaires à la société de masse a posé des problèmes nouveaux, qui ne se réduisent pas à l'idée de liens simples avec la pauvreté, et qui tiennent davantage aux transformations particulières subies par les relations sociales dans la nouvelle société émergente. Ce phénomène a été, en tant que tel, une nouvelle source de violence. Par ailleurs, l'effondrement des instruments de contrôle de la violence et les nouvelles formes de violence associées à la société de masse ont, ensemble, entraîné le Brésil dans une spirale, qui a été productrice d'un discours disposant d'une large écoute, un discours de légitimation de la violence auquel seuls les mouvements des droits de l'homme ont tenté de s'opposer. Envisager des sorties de la violence implique donc de prendre en considération chacun de ces éléments, pour essayer d'en inverser la logique.

Premièrement, une réforme s'impose au niveau des institutions chargées de la sécurité publique, notamment la police et la justice, de façon à passer de conditions de fonctionnement aujourd'hui fondées sur le couple violence/inefficacité, vers d'autres, davantage fondées sur le couple efficacité/emploi minimal de la violence. Ces deux éléments vont ensemble. Par ailleurs, il est difficile de concevoir une réforme de la police sans que la justice soit aussi impliquée, et sans que la redéfinition de leurs tâches respectives et des mécanismes de contrôle de la police par la justice ne soit prise en ligne de compte.

Deuxièmement, la sortie de la violence implique que soient radicalisées les conditions d'entrée des populations pauvres dans la société de masse, par exemple en complétant l'urbanisation des quartiers populaires, ou en améliorant encore plus, par le biais des politiques publiques, leur niveau de vie ; mais aussi, en prenant compte des problèmes spécifiques posés par cette nouvelle situation. Ainsi, un travail de reconstruction symbolique est plus que jamais nécessaire, en ce qui concerne l'image des populations noires, fondé sur une définition culturellement positive de ces populations, ce qui est à la base d'un nouveau modèle d'égalité sociale.

Enfin, pour inverser la tolérance à l'égard de la violence, qui s'est exprimée à tous les niveaux ces dernières années dans la société brésilienne, une volonté politique explicite devrait aider à construire une nouvelle capacité de la société brésilienne à se ressouder autour de la non-violence. A ce niveau, presque tout reste à faire, qu'il s'agisse de renforcer la capacité de refus de la violence du chef de l'Etat, en tant que représentant de la collectivité nationale, et dont la fermeté en ce domaine semble un élément essentiel, ou d'assurer le rétablissement symbolique, dans la même perspective, du rôle des élus politiques. Le mouvement Viva Rio, à Rio de Janeiro, a donné l'exemple, en créant un large rassemblement de personnalités politiques, entrepreneurs, journalistes 
et leaders communautaires autour d'activités susceptibles de favoriser la réduction de la violence. Mais cette expérience, pour l'instant isolée, devrait être développée à d'autres niveaux et dans bien d'autres régions du pays.

* Chargée de recherches au CADIS.

\section{OUVRAGES CITES}

Adorno Sergio, " A Criminalidade Urbana Violenta no Brasil : Um Recorte Tematico ", Rio de Janeiro, BIB, n 35, 1er semestre 1993, p. 3-24.

Akerman Marco (coord.), Mapa de Risco da Violência. Cidade de São Paulo, São Paulo, CEDEC, 1996.

Americas Watch, " Massacre na Casa de Detençao ", Os Direitos Humanos no Brasil, São Paulo, Nucleo de Estudos da Violência (Universidade de São Paulo) e Comissão Teotônio Vilela, 1993.

Americas Watch, Violência Policial Urbana no Brasil. Mortes e Tortura pela Policia em São Paulo e no Rio de Janeiro nos Ultimos Cinco Anos, 1987-1992. São Paulo, Nucleo de Estudos da Violência (Universidade de São Paulo), $1993 b$.

Amorim Carlos, Comando Vermelho. A historia secreta do crime organizado, Rio de Janeiro, Editora Record, 1993.

Barcellos Caco, Rota 66. A historia da policia que mata, São Paulo, Editora Globo, 1992.

Belloch Israel, Capa Preta e Lurdinha. Tenorio Cavalcanti e o Povo da Baixada, Rio de Janeiro, Editora Record, 1986.

Benevides Maria Victoria, " Linchamentos : violência e 'justiça' popular ", in Violência Brasileira, São Paulo, Brasiliense, 1982.

Benevides Maria Victoria, " Violência, povo e policia ", Violência urbana no noticiario de imprensa, São Paulo, Brasiliense 1983.

Bretas Marcos Luiz, " A historia e a crise das policias : observaçoes sobre a falência e a permanência dos modelos policiais ", Seminario Internacional " Estratégias de Intervençao Policial no Estado Democratico ", São Paulo, Nucleo de Estudos da Violência da Universidade de São Paulo, 2-4 setembro 1996.

Caldeira Cesar, " Seqüestros no Rio de Janeiro ", Seminario Internacional " Estratégias de Intervençao Policial no Estado Democratico ", São Paulo, Nucleo de Estudos da Violência da Universidade de São Paulo, 2-4 setembro 1996a.

Caldeira Cesar, "Operaçao Rio e Cidadania: as Tensoes entre o Combate à Criminalidade e a Ordem Juridica ", Politica e Cultura. Visoes do Passado e Perspectivas Contemporâneas, São Paulo, Hucitec/ANPOCS, 1996 b.

Caldeira Teresa Pires do Rio, " Direitos Humanos ou 'Privilégios de Bandidos'? Desventuras da democratizaçao brasileira " dans Novos Estudos, n 30, julho, 1991.

60 Caldeira Teresa Pires do Rio, City of Walls. Crime, Segregation and Citizenship in São Paulo. University of California at Berkeley, 1992.

1 Cordeiro Renato et Fagundes Renato, " Nilo Batista abre guerra contra Betinho ", Jornal do Brasil, 24 juillet 1996.

Da Matta Robert,. " As raizes da violência no Brasil ", dans (divers auteurs), Violência Brasileira, São Paulo, Brasiliense, 1982. 

Paris, Seuil, 1983. psychotropes, Paris, Editions Esprit, 1991. CTV, 1995. moderne, Paris, Fayard, 1966, chapitre II. Rio de Janeiro, Zahar, 1995. de São Paulo, 23.6.1996. agosto de 1989. Paulo, Scritta Editorial, 1992. Perspectivas, São Paulo, Vértice, 1988.

Da Matta Roberto, Carnavals, bandits et héros. Ambiguïtés de la société brésilienne,

Dubet François, La Galère. Jeunes en survie, Paris, Gallimard, 1987.

Ehrenberg Alain, Individus sous influence. Drogues, alcools, médicaments

Fase, Ibase, Idac, Iser, " Levantamento de meninas e meninos nas ruas do Rio de Janeiro ", Relatorio de pesquisa, Projeto Se essa rua fosse minha, Março de 1992.

Fernandes Heloisa Rodrigues, " Violência e Modos de vida: 'Os Justiceiros' ", dans Tempo Social, Revista de Sociologia. USP, São Paulo 4 (1-2) :43-52, 1992.

Godinho Fernando e Nogueira Rui, " Estudo mostra que so Colômbia é mais violenta do que o Brasil ", Folha de São Paulo, 18.3.1996.

Hanashiro Olaya, Sinhoretto Jacqueline e Singer Helena, " Linchamentos : a democracia mudou alguma coisa? ", dans Direitos Humanos no Brasil, n 2, São Paulo, NEV-USP e

Hobsbawm Eric, " Le bandit social ", dans Les primitifs de la révolte dans l'Europe

Lima Roberto Kant de, A Policia da Cidade do Rio de Janeiro : seus Dilemas e Paradoxos,

Lozano André et Schlegel Rogerio, " Assassinato apavora 63\% das pessoas ", dans Folha

Martins José de Souza, " Linchamentos : a vida por um fio ", dans Travessia, maio-

Mingardi Guaracy, Tiras, Gansos e Trutas. Cotidiano e Reforma na Policia Civil, São

Muniz Jacqueline, " Os direitos dos outros e outros direitos: um estudo sobre a negociaçao de conflitos nas DEAMs/RJ ", Rio de Janeiro, Iser/Relume Dumara, 1996.

7 Muniz Jacqueline, Larvie Patrick, Musumeci Leonarda et Freire Bianca, " Resistências e dificuldades na implantaçao do programa de Policiamento Comunitario em Copacabana, Rio de Janeiro ", Seminario Internacional " Estratégias de Intervençao Policial no Estado Democratico ", São Paulo, Nucleo de Estudos da Violência da Universidade de São Paulo, 2-4 setembro 1996.

Oliveira Paulo et als, " Série de reportagens sobre os exterminadores da Baixada Fluminense ", O Dia, 4, 5, 6, 7, 9, 10, 11 et 19 juillet 1993.

9 Paixão Antonio Luiz, " Crime, controle social e consolidação da democracia ", dans Reis Fabio Wanderley et O'Donnell Guillermo (org.), A Democracia no Brasil. Dilemas e

Peralva Angelina e Chamlian Helena Coharik, A relaçao professor-aluno no cotidiano escolar, Rapport de recherche, São Paulo, FEUSP, 1984.

Peralva Angelina, Reinventando a escola. A luta dos professores publicos de São Paulo na transiçao democratica, Tese de livre-docência em sociologia da educaçao, Faculdade de Educaçao, Universidade de São Paulo, 1992 (à paraître). 
Peralva Angelina, " L'expérience allemande ", dans Michel Wieviorka (ss la dir de) Racisme et xénophobie en Europe, Paris, La Découverte, 1994.

Peralva Angelina, " Démocratie et violence : un regard sur le cas de Rio de Janeiro ", dans Problèmes d'Amérique Latine, décembre 1996.

Paz Carlos Eugênio, Viagem à Luta Armada, Rio de Janeiro, Civilizaçao Brasileira, 1996.

Pereira Affonso e Oliveira Luciano, " A policia na boca do povo ", CPoli-Fundaj/ Ministério da Justiça - Programa Ruas em Paz. Recife, 1986.

Pinheiro Paulo Sergio, " Cronologia da violência - 1979-1983 ", dans Escritos Indignados, São Paulo, Brasiliense, 1984.

Pinheiro Paulo Sergio, " Autoritarismo e transição ", dans Revista USP, 9, março, abril, maio de 1991.

Pinheiro Paulo Sergio, Izumino Eduardo A. e Fernandes Maria Cristina Jakimik, " Violência Fatal : Conflitos Policiais em São Paulo ", dans Revista USP, 9, março, abril, maio de 1991.

Pinheiro Paulo Sergio, " São Paulo: People on the Margin and Civil Society ", dans Seminar Place and Right Conference, Ardem Homestead, New York, (sponsored by the Committee on Theory and Culture), September 11-13, 1992.

Pinheiro Paulo Sergio, " Direitos Humanos no Ano que Passou: Avanços e Continuidades ", dans Direitos Humanos no Brasil, n² 2, São Paulo, Núcleo de Estudos da Violência da Universidade de São Paulo e Comissão Teotônio Vilela, 1995.

Sigaud Lygia, " Milicias, Jagunços e Democracia ", dans Ciência Hoje, Suplemento, n 28, vol. 5, janeiro/fevereiro, 1987.

Silva Roberto, A criminalizaçao de uma geraçao de orfaos e abandonados, dissertaçao de mestrado. São Paulo, Faculdade de Educaçao, Universidade de São Paulo, 1996.

3 Silva Rose Neubauer e Mello Guiomar Namo, " Politica Educacional para os Anos 90 ", dans (divers auteurs) Estado e Educação, Campinas et São Paulo, Papirus/Cedes/Ande/ Anped, d1992.

4 Soares Barbara Musumeci, " Delegacia de atendimento à mulher : questao de gênero, numero e grau ", dans Violência e Politica no Rio de Janeiro, Rio de Janeiro, Iser/ Relume Dumara, 1996a. estudo sobre éticas populares e cultura politica ", dans Violência e Politica no Rio de Janeiro, Rio de Janeiro, Iser/Relume Dumara, 1996b.

Soares Luiz Eduardo, Milito Claudia e Silva Hélio R. S., " Homicidios dolosos praticados contra crianças e adolescentes, no Estado do Rio de Janeiro -1991 a Julho de 1993 ", dans Violência e Politica no Rio de Janeiro, Rio de Janeiro, Iser/Relume Dumara, 1996.

7 Soares Luiz Eduardo, Soares Barbara Musumeci et Carneiro Leandro Piquet, " Violência contra a mulher : as DEAMs e os pactos domésticos ", dans Violência e Politica no Rio de Janeiro, Rio de Janeiro, Iser/Relume Dumara, 1996c.

Soares Luiz Eduardo, Sé Joao Trajano Sento, Rodrigues José Augusto de Souza et Carneiro Leandro Piquet, " Criminalidade urbana e violência : o Rio de Janeiro no contexto internacional ", dans Violência e Politica no Rio de Janeiro, Rio de Janeiro, Iser/Relume Dumara, 1996d. 
Souza Percival de, A maior violência do mundo. Baixada Fluminense, Rio de Janeiro, Brasil, São Paulo, Traço Editora, 1980.

Sussekind Elizabeth, " A manipulaçao politica da criminalidade ", dans Ciência Hoje, volume 5, $n^{\circ} 28$. Suplemento Especial, janeiro/fevereiro 1987.

Tedrus Maria Aparecida Lealdini, Jovens: trabalho nas ruas e experiências de sociabilidade, Dissertaçao de mestrado. São Paulo, Faculdade de Educaçao, Universidade de São Paulo, 1996.

Toledo José Roberto de, " Em 15 anos, homicidios triplicam no Brasil ", Folha de São Paulo, 11.11.1996.

Velho Gilberto, Nobres e anjos, Um estudo sobre toxicos e hierarquia. São Paulo, Faculdade de Filosofia, Letras e Ciências Humanas, Universidade de São Paulo, 1975.

Velho Gilberto, " As vitimas preferenciais ", dans Ciência Hoje, volume 5, n 28. Suplemento Especial, janeiro/fevereiro 1987.

Weffort Francisco, " A América Errada ", Lua Nova, n² 21, São Paulo, setembro 1990.

Zanini Fabio, " Garoto de 14 anos é morto por policial ", dans Folha de São Paulo, 22.11.1996.

Revenir à la partie précédente

Revenir à la première partie

\section{NOTES}

1. Nous ne pourrons pas nous arrêter, dans cette étude, sur la question des menaces de déplacement qui pendant très longtemps ont pesé sur les populations des favelas.

Certaines populations habitant des favelas de la zone sud de Rio ont été effectivement déplacées vers des quartiers lointains, sous prétexte de leur offrir des logements meilleurs. Mais dans l'ensemble, les favelados ont montré une forte capacité de résistance et ils ont été soutenus par ceux qui prônaient des politiques d'urbanisation des favelas, plutôt que des politiques de déplacement des populations. Maintes favelas se sont effectivement urbanisées peu à peu, devenant de véritables quartiers populaires incrustés dans les quartiers riches. Ce mouvement a acquis une impulsion particulièrement importante au moment de la redémocratisation, lorsque la menace de déplacement a quasiment disparu.

2. Caldeira Teresa Pires do Rio, City of Walls..., op. cit.

3. Ce qui est surtout vrai pour certains mouvements syndicaux et en particulier pour le mouvement des enseignants, que j'ai étudié à São Paulo. Cf. Peralva Angelina, Reinventando a escola. A luta dos professores publicos de São Paulo na transiçao democratica, Tese de livre-docência em sociologia da educaçao, Faculdade de Educaçao, Universidade de São Paulo, 1992.

4. Caldeira Teresa Pires do Rio, City of Walls..., op. cit. 
5. Hobsbawm Eric, " Le bandit social ", dans Les primitifs de la révolte dans l'Europe moderne, Paris, Fayard, 1966, chapitre II.

6. Cette affirmation est de même nature que celle qui a été formulée, dans les années quatre-vingt-dix, par le gouvernement allemand à l'égard de la jeunesse allemande. On reconnaissait ainsi officiellement, dans cette tranche d'âge, " un potentiel de violence disponible à long terme ", qui n'était pas sans lien avec les transformations les plus récentes de la société allemande dans le courant de la décennie (Peralva, 1994).

7. Ehrenberg Alain, Individus sous influence. Drogues, alcools, médicaments psychotropes, Paris, Editions Esprit, 1991.

8. Velho Gilberto, Nobres e anjos, Um estudo sobre toxicos e hierarquia, São Paulo, Faculdade de Filosofia, Letras e Ciências Humanas, Universidade de São Paulo, 1975.

9. Alors qu'à São Paulo, une partie importante du marché consommateur du crack se développe dans les quartiers pauvres des périphéries urbaines, dans les favelas de Rio la consommation de cocaïne et d'herbe semble bien plus limitée. En général, seuls les jeunes mêlés au trafic fument des joints, les familles opposant une résistance ferme à leur consommation par les autres jeunes ; tous s'interdisent par ailleurs de consommer de la cocaïne.

10. Peralva Angelina, " Démocratie et violence : un regard sur le cas de Rio de Janeiro " in Problèmes d'Amérique Latine, décembre 1996.

INDEX

Mots-clés : violence, villes/ sociologie urbaine, démocratie

Index géographique : Amérique du Sud, Brésil 